\title{
Sensor Fault Tolerant Control of Induction Motors
}

\author{
María Seron* Mónica Romero ${ }^{1 * *}$ José De Doná* $^{*}$ \\ * Centre for Complex Dynamic Systems and Control, The University \\ of Newcastle, Callaghan NSW 2308, Australia (e-mails: maria.seron \\ and jose.dedona@newcastle.edu.au) \\ ** Laboratorio de Sistemas Dinámicos y Procesamiento de la \\ Información, Universidad Nacional de Rosario, Riobamba 245 bis, \\ 2000 Rosario, Argentina (e-mail:mromero@fceia.unr.edu.ar)
}

\begin{abstract}
In this paper we propose a multiobserver switching control strategy for fault tolerant control of induction motors. The strategy combines three current sensors and associated observers that estimate the rotor flux. The estimates provided by the observers are compared at each sampling time by a switching mechanism which selects the sensors-observer pair with the smallest error between the estimated flux magnitude and a desired flux reference. The selected estimates are used by a field oriented controller to implement the control law. Pre-checkable conditions are derived that guarantee fault tolerance under an abrupt fault of a current sensor. Simulation results under realistic conditions illustrate the effectiveness of the scheme.
\end{abstract}

\section{INTRODUCTION}

In this paper, we propose the use of a fault tolerant switching strategy that combines stator current sensors, rotor flux observers and a well-studied controller for induction motors. For the controller component of the scheme, we consider the field oriented control (FOC) technique for induction motors, first introduced by Blaschke (1972) and revisited in, for example, Marino et al. (1993). The implementation of this technique requires measurements or estimates of the rotor speed, stator currents and rotor flux. We will assume that the rotor speed and stator currents are measured. In contrast, the rotor flux is estimated from the available measurements by means of flux observers of the form proposed by Kubota and Matsuse (1994).

The structure of the proposed fault tolerant control scheme is depicted in Figure 1. It consists of a bank of three flux observers, an estimate switching mechanism and the FOC controller. Each observer provides estimates of the rotor flux based on noisy measurements of two phase currents. At each sampling time, the switching mechanism selects the observer with the smallest error between the estimated flux magnitude and a desired flux reference, and passes the selected state estimates to the FOC controller. The latter uses the selected state estimates in place of the (unavailable) true states to implement the control law.

A standard approach to achieve fault tolerance is to endow the control system with explicit fault detection and compensation capabilities (see, for example, Lee and Ryu (2003) for the use of this approach in induction motor control systems). In contrast, our proposed strategy comes with pre-checkable conditions which guarantee that, when a current sensor fails, the observers that use measurements from the faulty sensor are automatically avoided by the

\footnotetext{
1 This work was partly done while Monica Romero was on academic visit at LSIS, Université Paul Cezanne, Marseille, France
}

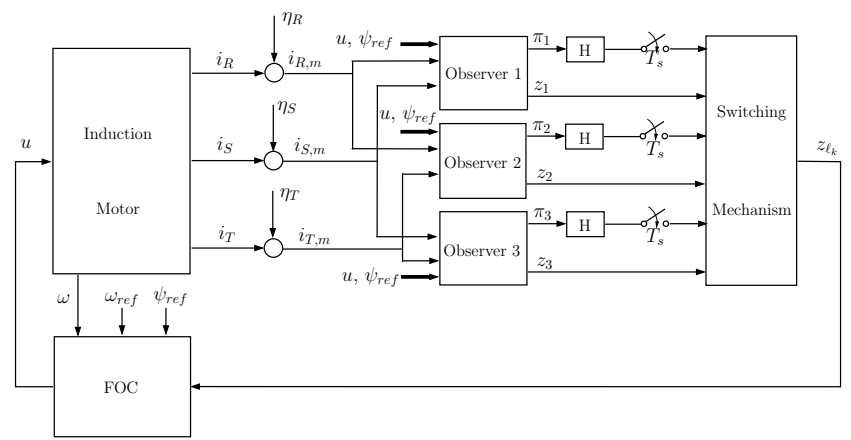

Fig. 1. Fault tolerant control scheme for the induction motor, including flux observers, estimate switching mechanism and field oriented control (FOC).

switching mechanism, thus maintaining good performance levels even under sensor fault. Thus, our scheme achieves faulty sensor detection and isolation "implicitly" by guaranteeing that the switching cost avoids selecting faulty sensors.

\section{MODEL OF THE INDUCTION MOTOR}

We consider the model of the induction motor in a reference frame fixed with the stator, with components denoted by $(a, b)$ (Krause et al., 1995). In this reference frame, the motor electromagnetic variables can be described by the following dynamic equation:

$$
\frac{d x}{d t}=A(\omega) x+B u, x=\left[i_{a} i_{b} \psi_{a} \psi_{b}\right]^{T}, u=\left[u_{a} u_{b}\right]^{T},
$$

where the state $x$ is composed by the stator current components $i_{a}, i_{b}$ and the rotor flux components $\psi_{a}, \psi_{b}$, and the input $u$ consists of the stator voltage components $u_{a}, u_{b}$. The matrices $A(\omega)$ and $B$ in (1) have the form 


$$
A=\left[\begin{array}{ll}
a_{r 11} \mathcal{I} & a_{r 12} \mathcal{I}+a_{i 12}(\omega) \mathcal{J} \\
a_{r 21} \mathcal{I} & a_{r 22} \mathcal{I}+a_{i 22}(\omega) \mathcal{J}
\end{array}\right], B=\left[\begin{array}{c}
\frac{1}{\sigma L_{s}} \mathcal{I} \\
0
\end{array}\right]
$$

where

$$
\begin{aligned}
& a_{r 11} \triangleq-\frac{R_{s}}{\sigma L_{s}}-\frac{1-\sigma}{\sigma \tau_{r}}, a_{r 12} \triangleq \frac{1}{c \tau_{r}}, a_{i 12}(\omega) \triangleq-\frac{n_{p} \omega}{c}, \\
& a_{r 21} \triangleq \frac{M}{\tau_{r}}, a_{r 22} \triangleq-\frac{1}{\tau_{r}}, a_{i 22}(\omega) \triangleq n_{p} \omega, \\
& \mathcal{I}=\left[\begin{array}{ll}
1 & 0 \\
0 & 1
\end{array}\right], \quad \mathcal{J}=\left[\begin{array}{cc}
0 & -1 \\
1 & 0
\end{array}\right],
\end{aligned}
$$

and $n_{p}$ is the number of pole pairs of the induction machine, $R_{s}, R_{r}, L_{s}, L_{r}$, are the stator and rotor resistances and self-inductances, respectively, $M$ is the mutual inductance, $\tau_{r}=L_{r} / R_{r}, \sigma=1-M^{2} /\left(L_{s} L_{r}\right)$ and $c=\sigma L_{s} L_{r} / M$. In addition, the rotor speed $\omega$ satisfies

$$
\frac{d \omega}{d t}=\frac{n_{p} M}{J L_{r}}\left[\psi_{a} i_{b}-\psi_{b} i_{a}\right]-\frac{\tau_{l}}{J}
$$

where $J$ is the moment of inertia of the rotor and $\tau_{l}$ is the load torque, which is assumed constant.

The stator current components $i_{a}, i_{b}$ in (1) are the twophase projection on the $(a, b)$ plane of the three-phase currents $i_{R}, i_{S}$ and $i_{T}$ (Krause et al., 1995):

$$
i_{a}=(1 / 3)\left(2 i_{R}-i_{S}-i_{T}\right), \quad i_{b}=(\sqrt{3} / 3)\left(i_{S}-i_{T}\right) .
$$

In Section 3 we will describe a control strategy that is a function of the states of system (1)-(4). To implement the control law, we will assume that both $w$ and $i_{R}, i_{S}, i_{T}$ are measured. Equations (5) then directly give the state variables $i_{a}$ and $i_{b}$. The remaining state variables $\psi_{a}$ and $\psi_{b}$ in (1) will be estimated by means of flux observers.

\section{FIELD ORIENTED CONTROL}

We will employ the classic field oriented control (FOC) technique first introduced by Blaschke (1972). Our presentation follows Marino et al. (1993). Defining $\rho=$ $\arctan \left(\psi_{b} / \psi_{a}\right)$, FOC uses the transformations

$\left[\begin{array}{l}i_{d} \\ i_{q}\end{array}\right]=R_{\rho}\left[\begin{array}{l}i_{a} \\ i_{b}\end{array}\right],\left[\begin{array}{l}\psi_{d} \\ \psi_{q}\end{array}\right]=R_{\rho}\left[\begin{array}{l}\psi_{a} \\ \psi_{b}\end{array}\right], R_{\rho} \triangleq\left[\begin{array}{cc}\cos \rho & \sin \rho \\ -\sin \rho & \cos \rho\end{array}\right]$.

We have from (6) that $\psi_{q}=0$ and

$$
\psi_{d}=\sqrt{\psi_{a}^{2}+\psi_{b}^{2}}
$$

Under (6) and the input transformation

$$
\left[\begin{array}{l}
u_{a} \\
u_{b}
\end{array}\right]=\sqrt{\psi_{a}^{2}+\psi_{b}^{2}}\left[\begin{array}{cc}
\psi_{a} & \psi_{b} \\
-\psi_{b} & \psi_{a}
\end{array}\right]^{-1}\left[\begin{array}{l}
u_{d} \\
u_{q}
\end{array}\right]
$$

the system (1)-(4) becomes

$$
\begin{aligned}
& \frac{d \psi_{d}}{d t}=-\frac{1}{\tau_{r}} \psi_{d}+\frac{M}{\tau_{r}} i_{d}, \\
& \frac{d i_{d}}{d t}=-\gamma i_{d}+\frac{\beta}{\tau_{r}} \psi_{d}+n_{p} \omega i_{q}+\frac{M}{\tau_{r}} \frac{i_{q}^{2}}{\psi_{d}}+\frac{1}{\sigma L_{s}} u_{d}, \\
& \frac{d i_{q}}{d t}=-\gamma i_{q}-\beta n_{p} \omega \psi_{d}-n_{p} \omega i_{d}-\frac{M}{\tau_{r}} \frac{i_{q} i_{d}}{\psi_{d}}+\frac{1}{\sigma L_{s}} u_{q}, \\
& \frac{d \omega}{d t}=\mu \psi_{d} i_{q}-\frac{\tau_{l}}{J}, \\
& \frac{d \rho}{d t}=n_{p} \omega+\frac{M}{\tau_{r}} \frac{i_{q}}{\psi_{d}},
\end{aligned}
$$

where $\gamma=M^{2} R_{r} /\left(\sigma L_{s} L_{r}^{2}\right)+R_{s} /\left(\sigma L_{s}\right), \beta=M /\left(\sigma L_{r} L_{s}\right)$ and $\mu=n_{p} M /\left(J L_{r}\right)$. The objectives of the FOC methodology are to regulate the rotor flux amplitude (7) to a constant reference value $\psi_{\text {ref }}$ and to have the rotor speed $\omega$ track a desired reference trajectory $\omega_{\text {ref }}$. These objectives are achieved by combining the nonlinear transformation

$$
\left[\begin{array}{l}
u_{d} \\
u_{q}
\end{array}\right]=\sigma L_{s}\left[\begin{array}{c}
-n_{p} \omega i_{q}-\frac{M}{\tau_{r}} \frac{i_{q}^{2}}{\psi_{d}}-\frac{\beta}{\tau_{r}} \psi_{d}+v_{d} \\
n_{p} \omega i_{d}+\frac{M}{\tau_{r}} \frac{i_{q} i_{d}}{\psi_{d}}+\beta n_{p} \omega \psi_{d}+v_{q}
\end{array}\right]
$$

and the PI controllers

$$
\begin{aligned}
& v_{d}=-k_{d 1}\left(\psi_{d}-\psi_{r e f}\right)-k_{d 2} \int_{0}^{t}\left(\psi_{d}(s)-\psi_{\text {ref }}\right) d s, \\
& v_{q}=-k_{q 1}\left(\tau_{e}-\tau_{r e f}\right)-k_{q 2} \int_{0}^{t}\left(\tau_{e}(s)-\tau_{r e f}(s)\right) d s, \\
& \tau_{r e f}=-k_{q 3}\left(\omega-\omega_{r e f}\right)-k_{q 4} \int_{0}^{t}\left(\omega(s)-\omega_{r e f}\right) d s,
\end{aligned}
$$

where $\psi_{\text {ref }}$ is the desired constant reference value for the flux amplitude, $\tau_{e}=\mu \psi_{d} i_{q}$ and $\omega_{\text {ref }}$ is the desired reference signal for the rotor speed. The resulting FOC controller is a function of the reference signals and of the states of system (9); moreover, through the transformations (6)$(8)$, it is also a function of the state $x=\left[i_{a} i_{b} \psi_{a} \psi_{b}\right]^{T}$ of system (1). We will denote this function as

$$
u=\mathcal{K}_{\mathrm{FOC}}\left(\omega, x, \omega_{\text {ref }}, \psi_{\text {ref }}\right)
$$

In Sections 4 and 5 below, we will describe the strategy used for measurement and estimation of the states required to implement the FOC law (14).

\section{CURRENT SENSORS AND FLUX OBSERVERS}

The phase currents $i_{R}, i_{S}, i_{T}$ satisfy the algebraic relation

$$
i_{R}+i_{S}+i_{T}=0 \text {. }
$$

Hence, if two phase currents are measured then the third phase current can be calculated from (15). However, we propose to employ three sensors measuring the three currents $i_{R}, i_{S}$ and $i_{T}$, and take advantage of the redundancy provided by these three measurements in the following observer based strategy for fault tolerant control.

The sensor measurement equations have the form

$$
\begin{gathered}
i_{R, m}=i_{R}+\eta_{R}, \\
i_{S, m}=i_{S}+\eta_{S}, \\
i_{T, m}=i_{T}+\eta_{T},
\end{gathered}
$$

where $\eta_{R}, \eta_{S}$ and $\eta_{T}$ are bounded measurement noises.

We will use three observers, each one based on measurements from two phases. Observer 1 uses measurements (16) and (17) from phases $R$ and $S$ and computes, based on (15) and (5),

$$
\begin{aligned}
& i_{T, 1}=-i_{R, m}-i_{S, m}, \\
& i_{a, 1}=(1 / 3)\left(2 i_{R, m}-i_{S, m}-i_{T, 1}\right), \\
& i_{b, 1}=(\sqrt{3} / 3)\left(i_{S, m}-i_{T, 1}\right) .
\end{aligned}
$$

Then, the values of $i_{a, 1}$ and $i_{b, 1}$ obtained in (19) are used in the observer dynamic equation

$$
\frac{d \hat{x}_{1}}{d t}=A(\omega) \hat{x}_{1}+B u+G(\omega)\left[\begin{array}{l}
\hat{i}_{a, 1}-i_{a, 1} \\
\hat{i}_{b, 1}-i_{b, 1}
\end{array}\right],
$$

where $\hat{x}_{1}=\left[\hat{i}_{a, 1} \hat{i}_{b, 1} \hat{\psi}_{a, 1} \hat{\psi}_{b, 1}\right]^{T}$ is the state estimate provided by observer 1 , and $A(\omega), B$ and $u$ are as in (1). The observer gain matrix $G(\omega)$ in (20) has the following form proposed by Kubota and Matsuse (1994):

$$
G(\omega)^{T}=\left[\begin{array}{cccc}
g_{1} & g_{2}(\omega) & g_{3} & g_{4}(\omega) \\
-g_{2}(\omega) & g_{1} & -g_{4}(\omega) & g_{3}
\end{array}\right]
$$


where $g_{1}=(K-1)\left(a_{r 11}+a_{r 22}\right), g_{2}(\omega)=(K-1) a_{i 22}(\omega)$ $g_{3}=\left(K^{2}-1\right)\left(a_{r 21}+a_{r 11} c\right)-c g_{1}, g_{4}(\omega)=-c g_{2}(\omega)$, and all remaining symbols are as defined in (3). This gain is such that for each fixed value of $\omega$, the eigenvalues of $A(\omega)+G(\omega) C$, with $C=[I 0]$, have negative real part and are proportional to those of $A(\omega)$ by a factor of $K>0$. The following output equation is associated with observer 1 :

$$
z_{1}=\left[i_{a, 1} i_{b, 1} \hat{\psi}_{a, 1} \hat{\psi}_{b, 1}\right]^{T} .
$$

The variable $z_{1}$ is the signal that observer 1 will make available to the controller whenever the switching mechanism selects this observer to implement the control law.

In a similar way, observer 2 uses measurements (16) and (18) from phases $R$ and $T$ in the following equations:

$$
\begin{aligned}
i_{S, 2} & =-i_{R, m}-i_{T, m}, \\
i_{a, 2} & =(1 / 3)\left(2 i_{R, m}-i_{S, 2}-i_{T, m}\right), \\
i_{b, 2} & =(\sqrt{3} / 3)\left(i_{S, 2}-i_{T, m}\right), \\
\frac{d \hat{x}_{2}}{d t} & =A(\omega) \hat{x}_{2}+B u+G(\omega)\left[\begin{array}{l}
\hat{i}_{a, 2}-i_{a, 2} \\
\hat{i}_{b, 2}-i_{b, 2}
\end{array}\right], \\
z_{2} & =\left[i_{a, 2} i_{b, 2} \hat{\psi}_{a, 2} \hat{\psi}_{b, 2}\right]^{T},
\end{aligned}
$$

where all symbols are defined accordingly. Finally, observer 3 uses measurements (17) and (18) from phases $S$ and $T$ in the following equations:

$$
\begin{aligned}
i_{R, 3} & =-i_{S, m}-i_{T, m}, \\
i_{a, 3} & =(1 / 3)\left(2 i_{R, 3}-i_{S, m}-i_{T, m}\right), \\
i_{b, 3} & =(\sqrt{3} / 3)\left(i_{S, m}-i_{T, m}\right), \\
\frac{d \hat{x}_{3}}{d t} & =A(\omega) \hat{x}_{3}+B u+G(\omega)\left[\begin{array}{l}
\hat{i}_{a, 3}-i_{a, 3} \\
\hat{i}_{b, 3}-i_{b, 3}
\end{array}\right], \\
z_{3} & =\left[i_{a, 3} i_{b, 3} \hat{\psi}_{a, 3} \hat{\psi}_{b, 3}\right]^{T} .
\end{aligned}
$$

In the following section we will describe a mechanism to switch between the above three observers according to a selection criterion. The observer that achieves the best value of the criterion will pass its output $\left(z_{1}, z_{2}\right.$ or $\left.z_{3}\right)$ to be used as substitute for the (unavailable) true state $x$ in the FOC law (14).

\section{ESTIMATE SWITCHING MECHANISM AND CONTROL IMPLEMENTATION}

For each observer we consider the following error signal:

$$
\pi_{j} \triangleq\left|\hat{\psi}_{a, j}^{2}+\hat{\psi}_{b, j}^{2}-\psi_{\text {ref }}^{2}\right|, \quad j=1,2,3,
$$

which measures the deviation of the square of the corresponding estimate of the flux amplitude $\hat{\psi}_{d, j}^{2} \triangleq \hat{\psi}_{a, j}^{2}+\hat{\psi}_{b, j}^{2}$ from the desired squared reference $\psi_{\text {ref }}^{2}$.

As we will show in Section 6 below, in permanent regime and healthy operation the error signals (25) have small values proportional to the current sensor noises. However, when a current sensor fails, the observers that take measurements from that sensor produce error signals (25) which are (noisy) periodic signals with mean values larger than in healthy operation. This discrepancy between the mean values of the error signals in healthy and in faulty operation motivates us to pass each signal $\pi_{j}, j=1,2,3$, through a low pass filter with transfer function

$$
H(s)=\frac{1}{T_{H} s+1} .
$$

The parameter $T_{H}>0$ in (26) will be chosen so that all harmonic components of the error signals are sufficiently attenuated and the resulting filtered signals $h * \pi_{j}$ (here $h$ is the impulse response of the filter (26) and ' $*$ ' denotes convolution) essentially represent the mean value of $\pi_{j}$.

The filtered error signals $h * \pi_{j}$ are subsequently sampled with period $T_{s}$ to obtain the discrete-time signals

$$
\pi_{j}^{0}(k)=\left(h * \pi_{j}\right)\left(k T_{s}\right), \quad k=0,1, \ldots,
$$

for $j=1,2,3$.

Finally, the (filtered and sampled) error signals $\pi_{j}^{0}(k)$ are compared at each sampling time $k$ according to the following switching criterion:

$$
\ell_{k}=\operatorname{argmin}_{j}\left\{\pi_{j}^{0}(k): j \in\{1,2,3\}\right\} .
$$

At each sampling time $k$, then, the observer with index $\ell_{k}$ computed from (28) is selected by the switching mechanism and its output $z_{\ell_{k}}(t)$ passed on to the controller for $k T_{s} \leq t<(k+1) T_{s}$ to implement the FOC law (14). Thus, the controller is implemented in continuous time in the following way:

$$
u=\mathcal{K}_{\mathrm{FOC}}\left(\omega, z_{\ell_{k}}, \omega_{\text {ref }}, \psi_{\text {ref }}\right) .
$$

When compared with standard FOC based on the use of a single observer, the proposed multi-observer switching strategy has similar performance under healthy operation of all sensors and, more importantly, it has the advantage of preserving good performance levels under sensor outage. These properties will be analysed in the following sections and illustrated by a simulation example in Section 8 .

\section{PERFORMANCE UNDER HEALTHY OPERATION}

In this section we will analyse the performance of the scheme of Figure 1 in permanent regime and when all current sensors are operational.

Induction motor variables. In permanent regime the rotor speed and the flux magnitude reach the constant values $\omega=\omega_{\text {ref }}$ and $\psi_{d}=\psi_{\text {ref }}$. Substituting the latter in (9) and setting $d \psi_{d} / d t=0$ and $d \omega / d t=0$ in (9a) and $(9 \mathrm{~d})$, respectively, we obtain

$$
\begin{aligned}
& i_{d}=\frac{\psi_{\text {ref }}}{M}, \\
& i_{q}=\frac{\tau_{l}}{J \mu \psi_{\text {ref }}} .
\end{aligned}
$$

To retrieve the variables in the $(a, b)$-frame, we substitute $(31)$ in $(9 \mathrm{e})$ and integrate from $\rho(0)=\rho_{0}$ to obtain

$$
\rho(t)=\omega_{\rho} t+\rho_{0}, \quad \omega_{\rho} \triangleq n_{p} \omega_{r e f}+\frac{M}{\tau_{r}} \frac{\tau_{l}}{J \mu \psi_{r e f}^{2}} .
$$

Then, using (6) and (32) yields

$$
\begin{aligned}
& \psi_{a}(t)=\psi_{\text {ref }} \cos \left(\omega_{\rho} t+\rho_{0}\right), \\
& \psi_{b}(t)=\psi_{\text {ref }} \sin \left(\omega_{\rho} t+\rho_{0}\right) .
\end{aligned}
$$

Similarly, using (30)-(32) and (6) we obtain, after some trigonometric manipulations,

$$
\begin{aligned}
& i_{a}(t)=I_{a b} \sin \left(\omega_{\rho} t+\rho_{0}+\rho_{a b}\right), \\
& i_{b}(t)=-I_{a b} \cos \left(\omega_{\rho} t+\rho_{0}+\rho_{a b}\right),
\end{aligned}
$$

where $I_{a b}=\sqrt{\frac{\psi_{r e f}^{2}}{M^{2}}+\frac{\tau_{l}^{2}}{J^{2} \mu^{2} \psi_{r e f}^{2}}}$ and $\rho_{a b}=-\arctan \frac{J \mu \psi_{r e f}^{2}}{M \tau_{l}}$. Finally, the phase currents $i_{R}, i_{S}$ and $i_{T}$ can be obtained from (5), (15) and (34). For example, we have

$$
i_{R}(t)=i_{a}(t)=I_{a b} \sin \left(\omega_{\rho} t+\rho_{0}+\rho_{a b}\right),
$$


The above expressions for the induction motor variables in permanent regime will be used in Section 7 to analyse the performance of the multiobserver switching system under faulty operation and to derive fault tolerance conditions.

Observer variables. To analyse the observer variables in permanent regime, we define the estimation errors as

$$
\tilde{x}_{j}=\left[\tilde{i}_{a, j} \tilde{i}_{b, j} \tilde{\psi}_{a, j} \tilde{\psi}_{b, j}\right]^{T} \triangleq x-\hat{x}_{j}, \quad j=1,2,3,
$$

where $x$ is the state of the system (1) and $\hat{x}_{j}$ is the state estimate provided by observer $j$, for $j=1,2,3$. It is easy to show using (1) and the observer equations (19), (20), (23) and (24) that, under healthy operation of all current sensors, the estimation errors (36) satisfy

$$
\dot{\tilde{x}}_{j}=[A(\omega)+G(\omega) C] \tilde{x}_{j}+G(\omega) \eta_{j}, \quad j=1,2,3,
$$

where, for each fixed $\omega$, the matrix $A(\omega)+G(\omega) C$ is stable by design (see discussion after (21)) and $\eta_{1}=$ $\left[\begin{array}{ll}\eta_{R} & \frac{\sqrt{3}}{3}\left(\eta_{R}+2 \eta_{S}\right)\end{array}\right]^{T}, \eta_{2}=\left[\begin{array}{ll}\eta_{R} & -\frac{\sqrt{3}}{3}\left(\eta_{R}+2 \eta_{T}\right)\end{array}\right]^{T}$ and $\eta_{3}=\left[-\left(\eta_{S}+\eta_{T}\right)-\frac{\sqrt{3}}{3}\left(\eta_{T}-\eta_{S}\right)\right]^{T}$.

When $\omega=\omega_{\text {ref }}$ in permanent regime, since the noises $\eta_{j}, j=1,2,3$ are bounded by assumption, the states of (37) will be ultimately bounded. In particular, using a straightforward modification of Theorem 1 in Kofman et al. (2007), we can obtain the following result on ultimate bounds on the flux estimation errors.

Lemma 1. Let the noises be elementwise ${ }^{2}$ bounded as $\left|\eta_{j}\right| \leq \bar{\eta}_{j}, j=1,2,3$, for some vectors $\bar{\eta}_{j}$ with positive elements. Let $V \Lambda V^{-1}$ be the Jordan canonical form of the matrix $A\left(\omega_{r e f}\right)+G\left(\omega_{r e f}\right) C$. Then the flux estimation errors are elementwise ultimately bounded as

$$
\left|\tilde{\psi}_{a, j}\right| \leq \epsilon_{a, j}, \quad\left|\tilde{\psi}_{b, j}\right| \leq \epsilon_{b, j},
$$

for $j=1,2,3$, where

$$
\begin{aligned}
& \epsilon_{a, j} \triangleq\left[\begin{array}{llll}
0 & 0 & 1 & 0
\end{array}\right]|V|\left|\mathbb{R e}(\Lambda)^{-1}\right|\left|V^{-1} G\left(\omega_{r e f}\right)\right| \bar{\eta}_{j} \\
& \epsilon_{b, j} \triangleq\left[\begin{array}{llll}
0 & 0 & 0 & 1
\end{array}\right]|V|\left|\mathbb{R e}(\Lambda)^{-1}\right|\left|V^{-1} G\left(\omega_{r e f}\right)\right| \bar{\eta}_{j} .
\end{aligned}
$$

The next result uses Lemma 1 to obtain ultimate bounds for the observer error signals (25).

Lemma 2. Under the conditions of Lemma 1 and in permanent regime $\left(\psi_{d}^{2}=\psi_{\text {ref }}^{2}\right)$, the observer error signals (25) satisfy, for $j=1,2,3$,

$$
\pi_{j} \leq \bar{\pi}_{j}, \quad \bar{\pi}_{j} \triangleq \epsilon_{a, j}^{2}+\epsilon_{b, j}^{2}+2 \psi_{\text {ref }} \epsilon_{a, j}+2 \psi_{\text {ref }} \epsilon_{b, j} .
$$

Proof. First, using the fact that $\psi_{d}^{2}=\psi_{a}^{2}+\psi_{b}^{2}=\psi_{\text {ref }}^{2}$ in permanent regime, we can write

$$
\begin{aligned}
\pi_{j} & =\left|\hat{\psi}_{a, j}^{2}+\hat{\psi}_{b, j}^{2}-\psi_{r e f}^{2}\right| \\
& =\left|\tilde{\psi}_{a, j}^{2}+\tilde{\psi}_{b, j}^{2}-2 \psi_{a} \tilde{\psi}_{a, j}-2 \psi_{b} \tilde{\psi}_{b, j}\right|,
\end{aligned}
$$

for $j=1,2,3$. Thus,

$$
\pi_{j} \leq\left|\tilde{\psi}_{a, j}\right|^{2}+\left|\tilde{\psi}_{b, j}\right|^{2}+2\left|\psi_{a}\right|\left|\tilde{\psi}_{a, j}\right|+2\left|\psi_{b}\right|\left|\tilde{\psi}_{b, j}\right|
$$

Using Lemma 1 and $\left|\psi_{a}\right| \leq \psi_{\text {ref }},\left|\psi_{b}\right| \leq \psi_{\text {ref }}$, yields (40).

Finally, we use Lemma 2 to derive bounds on the filtered and sampled error signal $\pi_{j}^{0}(k), j=1,2,3$, defined in (27), on which the switching strategy (28) bases its decision at each sampling time. Indeed, noting that $H(s)$ in $(26)$ is a first order transfer function such that $H(0)=1$ (and hence upper bounds on its output signal are less than or

\footnotetext{
$2|M|$ and $\mathbb{R e}(M)$ indicate the elementwise magnitude and real part respectively, of a (possibly complex) matrix (vector) $M$.
}

equal to upper bounds on its input signal), we can bound $\pi_{j}^{0}(k), j=1,2,3$, in $(27)$ as

$$
\pi_{j}^{0} \leq \bar{\pi}_{j}, \quad \bar{\pi}_{j} \triangleq \epsilon_{a, j}^{2}+\epsilon_{b, j}^{2}+2 \psi_{r e f} \epsilon_{a, j}+2 \psi_{r e f} \epsilon_{b, j},
$$

where $\epsilon_{a, j}$ and $\epsilon_{b, j}$ are defined in (39). If the bounds on the noises are small, then the bounds (41) on the (filtered and sampled) observer error signals under healthy operation will also be small. As we will show in Section 7 below, this is in stark contrast with the bounds that these observer error signals have when a current sensor associated with the corresponding observer fails. This difference in bounds between healthy and faulty operation is the key to achieve fault tolerance in the proposed approach.

\section{PERFORMANCE UNDER CURRENT SENSOR FAULT AND FAULT TOLERANCE}

In this section we analyse the performance of the switching control scheme under abrupt faults of current sensors. We will model an abrupt fault as an instant change in the sensor measurement equations from (16)-(18) to

$$
i_{R, m}=\eta_{R}^{F}, \quad i_{S, m}=\eta_{S}^{F}, \quad i_{T, m}=\eta_{T}^{F},
$$

where $\eta_{R}^{F}, \eta_{S}^{F}$ and $\eta_{T}^{F}$ are bounded measurement noises. We will assume that only one sensor can fail at a time.

Following similar steps as in Seron et al. (2007), we will employ a circular argument to find conditions that guarantee robust performance when a sensor fails. The argument is based on the working hypothesis that, in the presence of a faulty sensor, only estimates provided by observers which take measurements from healthy sensors are selected by the switching mechanism. Under this working hypothesis, we will analyse, in the following two subsections, the variables relevant to the switching control scheme. This analysis will finally allow us to derive conditions that guarantee that the working hypothesis is satisfied.

Induction motor variables. Under the working hypothesis that only measurements from healthy sensors are used by the switching controller, the performance in permanent regime of the induction motor is not affected by a fault in a current sensor. Thus, all equations derived in Section 6 are still valid.

Observer variables. We will consider a fault modelled by (42) in the sensor that measures the phase current $i_{R}$ (a similar analysis can be performed for faults in the other phases). Note from (19), (23) and (24) that only observers 1 and 2 will be affected by this fault whereas observer 3 will remain unaffected.

Substituting the first equality of (42) in (19), (20) and (23) we have, after some calculations, that the estimation errors for observers 1 and 2 in permanent regime $\left(\omega=\omega_{e f}\right)$ change their dynamics from (37) to

$$
\dot{\tilde{x}}_{l}^{F}=(A+G C) \tilde{x}_{l}^{F}+G b_{l} i_{R}+G \eta_{l}^{F}, \quad l=1,2,
$$

where $\tilde{x}_{l}^{F}=\left[\tilde{i}_{a, l}^{F} \tilde{i}_{b, l}^{F} \tilde{\psi}_{a, l}^{F} \tilde{\psi}_{b, l}^{F}\right]^{T}$ denotes the "under-fault" estimation errors and where $A \triangleq A\left(\omega_{\text {ref }}\right), G \triangleq G\left(\omega_{\text {ref }}\right)$, $b_{1}=-\left[\begin{array}{ll}1 & \frac{\sqrt{3}}{3}\end{array}\right]^{T}, b_{2}=\left[\begin{array}{ll}-1 & \frac{\sqrt{3}}{3}\end{array}\right]^{T}, \eta_{1}^{F}=\left[\begin{array}{ll}\eta_{R}^{F} & \frac{\sqrt{3}}{3}\left(\eta_{R}^{F}+\right.\end{array}\right.$ $\left.\left.2 \eta_{S}\right)\right]^{T}$ and $\eta_{2}^{F}=\left[\begin{array}{ll}\eta_{R}^{F} & -\frac{\sqrt{3}}{3}\left(\eta_{R}^{F}+2 \eta_{T}\right)\end{array}\right]^{T}$.

Note that (43) is a stable linear system driven by two bounded external inputs: the phase current $i_{R}$ and the 
"under-fault" noise $\eta_{l}^{F}$. From (36) and taking Laplace transforms in (43) we have that the components of the flux estimation errors in response to $i_{R}$ satisfy

$$
\left[\begin{array}{c}
\tilde{\psi}_{a, l}^{F} \\
\tilde{\psi}_{b, l}^{F}
\end{array}\right]_{i_{R}}=\left[\begin{array}{c}
\tilde{H}_{a, l}(s) \\
\tilde{H}_{b, l}(s)
\end{array}\right] i_{R}
$$

where

$$
\begin{aligned}
\tilde{H}_{a, l}(s) & =\left[\begin{array}{llll}
0 & 0 & 1 & 0
\end{array}\right][s I-(A+G C)]^{-1} G b_{l}, \\
\tilde{H}_{b, l}(s) & =\left[\begin{array}{llll}
0 & 0 & 0 & 1
\end{array}\right][s I-(A+G C)]^{-1} G b_{l} .
\end{aligned}
$$

Since, in permanent regime, $i_{R}$ is a sine wave given by (35), then the flux estimation error components in (44) will also be sine waves of the form

$$
\left[\begin{array}{c}
\tilde{\psi}_{a, l}^{F} \\
\tilde{\psi}_{b, l}^{F}
\end{array}\right]_{i_{R}}=\left[\begin{array}{l}
\tilde{a}_{l} \sin \left(\omega_{\rho} t+\tilde{\rho}_{a, l}\right) \\
\tilde{b}_{l} \cos \left(\omega_{\rho} t+\tilde{\rho}_{b, l}\right)
\end{array}\right]
$$

where $\tilde{a}_{l}=\left|\tilde{H}_{a, l}\left(j \omega_{\rho}\right)\right| I_{a b}, \tilde{b}_{l}=\left|\tilde{H}_{b, l}\left(j \omega_{\rho}\right)\right| I_{a b}$, and where $\tilde{\rho}_{a, l}, \tilde{\rho}_{b, l}$ are some phase shifts.

Applying the principle of superposition to the system (43), assuming zero initial conditions, yields the following form for the flux estimation errors:

$$
\begin{gathered}
\tilde{\psi}_{a, l}^{F}=\tilde{a}_{l} \sin \left(\omega_{\rho} t+\tilde{\rho}_{a, l}\right)+\tilde{\eta}_{a, l}^{F}, \\
\tilde{\psi}_{b, l}^{F}=\tilde{b}_{l} \cos \left(\omega_{\rho} t+\tilde{\rho}_{b, l}\right)+\tilde{\eta}_{b, l}^{F},
\end{gathered}
$$

where we have combined (47) with the components of the flux estimation errors in response to the noise $\eta_{l}^{F}$, which we denote $\tilde{\eta}_{a, l}^{F}$ and $\tilde{\eta}_{b, l}^{F}$. These components are bounded as shown in the following result, which is similar to Lemma 1.

Lemma 3. Let the "under-fault" noises be elementwise bounded as $\left|\eta_{l}^{F}\right| \leq \bar{\eta}_{l}^{F}, l=1,2,3$, for some vectors $\bar{\eta}_{l}^{F}$ with positive elements. Let $V \Lambda V^{-1}$ be the Jordan canonical form of the matrix $A\left(\omega_{\text {ref }}\right)+G\left(\omega_{r e f}\right) C$. Then the components of the flux estimation errors in response to $\eta_{l}^{F}$ are elementwise ultimately bounded as

$$
\left|\tilde{\eta}_{a, l}^{F}\right| \leq \epsilon_{a, l}^{F}, \quad\left|\tilde{\eta}_{b, l}^{F}\right| \leq \epsilon_{b, l}^{F},
$$

for $l=1,2,3$, where

$$
\begin{gathered}
\epsilon_{a, l}^{F} \triangleq\left[\begin{array}{llll}
0 & 0 & 1 & 0
\end{array}\right]|V|\left|\mathbb{R e}(\Lambda)^{-1}\right|\left|V^{-1} G\left(\omega_{\text {ref }}\right)\right| \bar{\eta}_{l}^{F}, \\
\epsilon_{b, l}^{F} \triangleq\left[\begin{array}{llll}
0 & 0 & 0 & 1
\end{array}\right]|V|\left|\operatorname{Re}(\Lambda)^{-1}\right|\left|V^{-1} G\left(\omega_{\text {ref }}\right)\right| \bar{\eta}_{l}^{F} .
\end{gathered}
$$

We will use equations (48)-(51) to derive bounds for the observer error signals (25) under fault of the $R$ phase current sensor. The error signals (25) can be written (see the proof of Lemma 2) as

$$
\pi_{l}^{F}=\left|\left(\tilde{\psi}_{a, l}^{F}\right)^{2}+\left(\tilde{\psi}_{b, l}^{F}\right)^{2}-2 \psi_{a} \tilde{\psi}_{a, l}^{F}-2 \psi_{b} \tilde{\psi}_{b, l}^{F}\right|,
$$

where $\psi_{a}$ and $\psi_{b}$ satisfy (33). Using trigonometric relations and some manipulations, $\pi_{l}^{F}$ in (52) can be written as

$$
\pi_{l}^{F}=\left|\tilde{c}_{l}+\tilde{d}_{l} \sin \left(2 \omega_{\rho} t+\phi_{l}\right)+\tilde{e}_{l}\right|
$$

where

$$
\begin{array}{r}
\tilde{c}_{l}=\tilde{a}_{l}\left[\frac{\tilde{a}_{l}}{2}-\psi_{\text {ref }} \sin \left(\tilde{\rho}_{a, l}-\rho_{0}\right)\right]+ \\
\tilde{b}_{l}\left[\frac{\tilde{b}_{l}}{2}+\psi_{\text {ref }} \sin \left(\tilde{\rho}_{b, l}-\rho_{0}\right)\right],
\end{array}
$$

$$
\begin{gathered}
\tilde{d}_{l}^{2}=\psi_{r e f}^{2}\left[\tilde{a}_{l}^{2}+\tilde{b}_{l}^{2}+2 \tilde{a}_{l} \tilde{b}_{l} \cos \left(2 \rho_{0}+\tilde{\rho}_{a, l}+\tilde{\rho}_{b, l}\right)\right]+ \\
\frac{\tilde{a}_{l}^{4}}{4}+\frac{\tilde{b}_{l}^{4}}{4}-\frac{\tilde{a}_{l}^{2} \tilde{b}_{l}^{2}}{2} \cos \left(2 \tilde{\rho}_{a, l}+2 \tilde{\rho}_{b, l}-\pi\right), \\
\tilde{e}_{l}=\tilde{\eta}_{a, l}^{F}\left[\tilde{\eta}_{a, l}^{F}+2 \tilde{a}_{l} \sin \left(\omega_{\rho} t+\tilde{\rho}_{a, l}\right)-2 \psi_{r e f} \cos \left(\omega_{\rho} t+\rho_{0}\right)\right] \\
+\tilde{\eta}_{b, l}^{F}\left[\tilde{\eta}_{b, l}^{F}+2 \tilde{b}_{l} \cos \left(\omega_{\rho} t+\tilde{\rho}_{b, l}\right)-2 \psi_{\text {ref }} \sin \left(\omega_{\rho} t+\rho_{0}\right)\right],
\end{gathered}
$$

and where $\phi_{l}$ is some phase shift. The signal (53) can be lower bounded as

$$
\pi_{l}^{F} \geq g_{l}-\left|\tilde{e}_{l}\right|, \quad g_{l} \triangleq\left|\tilde{c}_{l}+\tilde{d}_{l} \sin \left(2 \omega_{\rho} t+\phi_{l}\right)\right| .
$$

Using (56) and the bounds derived in Lemma 3, a bound on $\left|\tilde{e}_{l}\right|$ can be computed as

$$
\left|\tilde{e}_{l}\right| \leq \epsilon_{a, l}^{F}\left(\epsilon_{a, l}^{F}+2 \tilde{a}_{l}+2 \psi_{\text {ref }}\right)+\epsilon_{b, l}^{F}\left(\epsilon_{b, l}^{F}+2 \tilde{b}_{l}+2 \psi_{\text {ref }}\right) \text {. }
$$

The term $g_{l}$ in (57) can be expressed as a Fourier series consisting of the sum of a mean value and harmonics of frequencies greater or equal to $2 \omega_{\rho}$. It can be shown that lower bound on the mean value, attained for $\tilde{c}_{l}=0$, is

$$
\operatorname{mean}\left(g_{l}\right) \geq \frac{2}{\pi}\left|\tilde{d}_{l}\right| \geq \frac{2}{\pi}\left|\tilde{a}_{l}-\tilde{b}_{l}\right| \sqrt{\psi_{r e f}^{2}+\frac{\left(\tilde{a}_{l}+\tilde{b}_{l}\right)^{2}}{4}},
$$

where the second inequality was obtained using worst cases for sines and cosines in (55). Combining (57), (58) and (59), we obtain

$$
\pi_{l}^{F} \geq \bar{\pi}_{l}^{F}+\left(2 \omega_{\rho} \text {-harmonic-terms }\right),
$$

where

$$
\begin{aligned}
& \bar{\pi}_{l}^{F} \triangleq \frac{2}{\pi}\left|\tilde{a}_{l}-\tilde{b}_{l}\right| \sqrt{\psi_{\text {ref }}^{2}+\frac{\left(\tilde{a}_{l}+\tilde{b}_{l}\right)^{2}}{4}}- \\
& \epsilon_{a, l}^{F}\left(\epsilon_{a, l}^{F}+2 \tilde{a}_{l}+2 \psi_{\text {ref }}\right)-\epsilon_{b, l}^{F}\left(\epsilon_{b, l}^{F}+2 \tilde{b}_{l}+2 \psi_{\text {ref }}\right) .
\end{aligned}
$$

The error signals (53), corresponding to a failed $\mathrm{R}$ phase current sensor, are filtered using $H(s)$ in $(26)$ and then sampled with period $T_{s}$ to produce the signals $\left(\pi_{l}^{F}\right)^{0}(k)$, $l=1,2$, defined as in (27), on which the switching strategy (28) bases its decision at each sampling time. Since $H(s)$ is a first order system with positive and monotonic impulse response, and the sampling operation preserves bounds, then the right hand side of (60) is also a lower bound for the (filtered and sampled) signals $\left(\pi_{l}^{F}\right)^{0}$. Moreover, if one chooses $T_{H}$ in (26) such that

$$
T_{H} \gg 1 /\left(2 \omega_{\rho}\right)
$$

then the filter sufficiently attenuates the $2 \omega_{\rho}$-harmonicterms, so that a lower bound for the signals $\left(\pi_{l}^{F}\right)^{0}$ can be approximated with arbitrary accuracy by

$$
\left(\pi_{l}^{F}\right)^{0} \geq \bar{\pi}_{l}^{F}, \quad l=1,2,
$$

with $\bar{\pi}_{l}^{F}$ as in (61).

Then, the scheme with switching criterion (28) will be fault tolerant under a fault of the phase $\mathrm{R}$ sensor if

$$
\bar{\pi}_{l}^{F}>\bar{\pi}_{3}, \quad \text { for } \quad l=1,2,
$$

where $\bar{\pi}_{l}^{F}$ and $\bar{\pi}_{3}$ were defined in (61) and (41), respectively. Note that, if condition (64) holds, then observer 3 will be chosen by the switching criterion (28) over observers 1 and 2, which are the ones affected by the faulty sensor measurements. Finally, similar conditions can be derived for faults in the two other phase current sensors.

\section{SIMULATIONS}

In this section we present simulation results for the control system of Figure 1. The parameters of the induction motor 

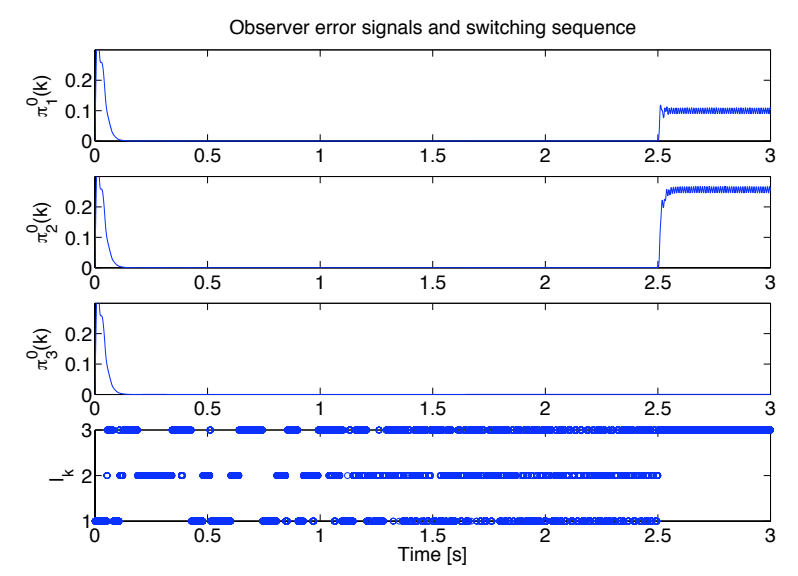

Fig. 2. Observer (filtered and sampled) error signals and switching sequence.

are: $R_{r}=0.39923 \Omega, R_{s}=1.165 \Omega, J=0.0812 \mathrm{Nm}$, $L_{s}=0.13995 \mathrm{Hy}, L_{r}=0.13995 \mathrm{Hy}, M=0.13421 \mathrm{Hy}$ and $n_{p}=2$. The desired constant reference values for the rotor speed and for the flux amplitude are $\omega_{\text {ref }}=154 \mathrm{rad} / \mathrm{s}$ and $\psi_{\text {ref }}=0.888 \mathrm{~Wb}$, respectively. The parameters for the PI controllers of the FOC strategy are $k_{d 1}=522.39$ and $k_{d 2}=1490.2$ in (11), $k_{q 3}=9.4081$ and $k_{q 4}=470.76$ in (13), and $k_{q 1}=2.9657$ and $k_{q 2}=449.78$ in (12) The sensor noises in (16)-(18) are bounded as $\left|\eta_{R}\right| \leq 9 \mathrm{~mA}$, $\left|\eta_{S}\right| \leq 9 \mathrm{~mA}$ and $\left|\eta_{T}\right| \leq 9 \mathrm{~mA}$. The same bound of $9 \mathrm{~mA}$ is used for the "under-fault" noises in (42). The observer gain parameter in (21) is chosen as $K=2$. For a load torque $\tau_{l}=30 \mathrm{Nm}$, the electric frequency $\omega_{\rho}$ in (32) takes the value $\omega_{\rho}=315.6 \mathrm{rad} / \mathrm{s}$. Thus, $1 /\left(2 \omega_{\rho}\right)=0.0016 \mathrm{~s}$ and we chose the filter parameter $T_{H}=0.0143 \mathrm{~s}$ in (26) so that (62) is satisfied. The switching sampling period used to update the selected observer in $(28)$ is $T_{s}=0.1 \mathrm{~ms}$. The fault tolerance conditions (64) are satisfied for the above parameters since $\bar{\pi}_{3}=0.0064$ and $\bar{\pi}_{1}^{F}=0.0426>\bar{\pi}_{3}$, $\bar{\pi}_{2}^{F}=0.0287>\bar{\pi}_{3}$. Thus, under a fault in the sensor that measures the phase current $i_{R}$, the scheme is guaranteed to choose only observer 3 , which is unaffected by the fault.

The simulation scenario is as follows. The reference signal for rotor speed is a ramp that starts from zero at $t=0 \mathrm{~s}$ and reaches its desired constant value $\omega_{\text {ref }}=154 \mathrm{rad} / \mathrm{s}$ at $t=2 \mathrm{~s}$. At $t=1 \mathrm{~s}$ a load with $\tau_{l}=30 \mathrm{Nm}$ is applied. At $t=2.5 \mathrm{~s}$ a fault in the sensor that measures the phase current $i_{R}$ occurs, that is, its measurement equation changes from (16) to (42). The top 3 plots of Figure 2 show the (filtered and sampled) error signals $\pi_{j}^{0}(k), j=1,2,3$, defined in (27), corresponding to observers 1, 2 and 3, respectively. As analysed in Section 7 , after the fault at $t=$ $2.5 \mathrm{~s}$ the error signals for observers 1 and 2 quickly move to values noticeably away from zero, whereas the error signal for observer 3 maintains the same small values (near zero) as before the occurrence of the fault. The bottom plot of Figure 2 shows the switching signal $\ell_{k}$ resulting from the switching mechanism decision (28). Note that after the fault at $t=2.5 \mathrm{~s}$ the switching mechanism only selects observer 3 , as guaranteed by the fault tolerance conditions (64). Figure 3 shows the response of the rotor flux magnitude $\psi_{d}$ (top) and the rotor speed $\omega$ (bottom) under the switching control scheme. Note that the fault at $t=2.5 \mathrm{~s}$ has no impact on these responses. Although not
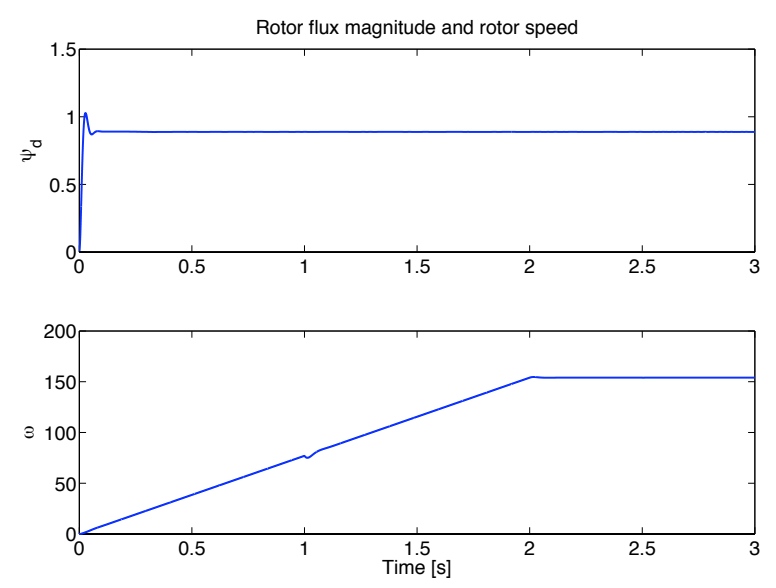

Fig. 3. Magnitude of the rotor flux and rotor speed under the switching control scheme.

shown in this example, a similar situation in terms of the bounds (64) for faults in phases $\mathrm{S}$ and $\mathrm{T}$ holds true. Thus, the scheme correctly selects the appropriate observer in the event of a fault in any of the phase current sensors, provided only one sensor fails at a time.

\section{CONCLUSIONS}

In this paper we have proposed the use of a multiobserver switching control strategy for sensor fault tolerant control of induction motors. The proposed strategy combines stator current sensors, rotor flux observers and a switching mechanism that selects the sensors-observer pair with the smallest error between the estimated flux magnitude and a desired flux reference. The estimates provided by the selected pair are used by a field oriented controller to implement the control law. We have provided precheckable conditions that guarantee fault tolerance under an abrupt fault of a current sensor. The results have been illustrated by a simulation example.

\section{REFERENCES}

F. Blaschke. The principle of field orientation applied to the new transvector closed-loop control system for rotating field machines. Siemens-Rev, 39:217-220, 1972.

E.J. Kofman, H. Haimovich, and M.M. Seron. A systematic method to obtain ultimate bounds for perturbed systems. International Journal of Control, 80(2):167178, February 2007.

P.C. Krause, O. Wasynczuk, and S.D Sudhoff. Analysis of Electric Machinery. IEEE press, New York, 1995.

H. Kubota and K. Matsuse. Speed sensorless field-oriented control of induction motor with rotor resistance adaptation. IEEE Trans. on Ind. Appl., 30(5):1219-1224, 1994.

K.-S. Lee and J.-S Ryu. Instrument fault detection and compensation scheme for direct torque controlled induction motor drives. IEE Proceedings. Control Theory and Applications, 150(4), July 2003.

R. Marino, S. Peresada, and P. Valigi. Adaptive inputoutput linearizing control of induction motors. IEEE Trans. on Aut. Contr., 38(2):208-221, 1993.

M.M. Seron, X.W. Zhuo, J.A. De Doná, and J.J. Martínez. Multisensor switching control strategy with fault tolerance guarantees. Automatica, 2007. To appear. 